\title{
A atuação do enfermeiro no Alojamento Conjunto na promoção do aleitamento
}

\section{materno}

\author{
The role of nurses in Rooming-in in promoting breastfeeding
}

El papel de las enfermeras en el Alojamiento Conjunto en la promoción de la lactancia materna

\begin{abstract}
Resumo
O Alojamento Conjunto é o lugar em que a mulher e o recém-nascido sadio, após o nascimento, permanecem em tempo integral juntos, até a alta hospitalar. Um dos benefícios do Alojamento Conjunto está na possibilidade do profissional de enfermagem estimular o fortalecimento do autocuidado e dos cuidados com recém-nascido, através de atividades de educação em saúde desenvolvidas durante a assistência prestada no período de internação do bionômio mãe/filho. Levando em considerações as recomendações dos Órgãos de Saúde à respeito da importância do aleitamento materno, é imprescindível que a assistência prestada no Alojamento Conjunto envolva orientações e auxílio a puérpera na prática de amamentação. Trata-se de um relato de experiência desenvolvido durante as práticas da disciplina de Estágio Supervisionado do curso de graduação de enfermagem, no Alojamento Conjunto de um hospital localizado em um município de médio porte na região Central do Estado do Rio Grande do Sul. O início da assistência prestada à puérpera precisa acontecer ainda no ambiente hospitalar, isto é, no Alojamento Conjunto, onde se reconhecem as primeiras modificações como estresse do parto, dores, início do processo de amamentação, medo, insegurança, dependência e sentimentos de ambivalência. Objetivou-se com esse trabalho relatar a experiência de uma enfermeira durante sua formação acadêmica, sobre a importância da assistência de enfermagem puerperal desenvolvida no Alojamento Conjunto direcionada no cuidado do binômio mãe/filho.
\end{abstract}

Palavras-chave: Enfermagem; Alojamento conjunto; Aleitamento materno.

\begin{abstract}
The Joint Accommodation is the place where the woman and the healthy newborn, after birth, remain full time together, until hospital discharge. One of the benefits of Joint Accommodation is the possibility for the nursing professional to encourage the strengthening of self-care and newborn care, through health education activities developed during the assistance provided during the hospitalization period of the mother / child. Taking into account the recommendations of the Health Organs regarding the importance of breastfeeding, it is essential that the assistance provided in the Joint Accommodation involves guidance and assistance to the puerperal woman in the practice of breastfeeding. This is an experience report developed during the practices of the Supervised Internship discipline of the undergraduate nursing course, in the Joint Accommodation of a hospital located in a medium-sized municipality in the Central region of the State of Rio Grande do Sul. the assistance provided to the puerperal woman still needs to happen in the hospital environment, that is, in the Joint Accommodation, where the first changes are recognized, such as labor stress, pain, the beginning of the breastfeeding process, fear, insecurity, dependence and feelings of ambivalence. The objective of this work was to report the experience of a nurse during her academic training, on the importance of puerperal nursing care developed in the Joint Accommodation directed to the care of the mother / child binomial.
\end{abstract}

Keywords: Nursing; Joint accommodation; Breastfeeding.

\section{Resumen}

La acomodación conjunta es el lugar donde la mujer y el recién nacido sano, después del nacimiento, permanecen juntos a tiempo completo hasta el alta hospitalaria. Uno de los beneficios del Alojamiento Conjunto es la posibilidad que tiene el profesional de enfermería de incentivar el fortalecimiento del autocuidado y el cuidado del recién nacido, 
a través de actividades de educación en salud desarrolladas durante la asistencia brindada durante el período de hospitalización de la madre / niño. Teniendo en cuenta las recomendaciones de los Órganos de Salud sobre la importancia de la lactancia materna, es fundamental que la asistencia brindada en el Alojamiento Conjunto involucre la orientación y asistencia a la puérpera en la práctica de la lactancia materna. Se trata de un relato de experiencia desarrollado durante las prácticas de la disciplina Pasantía Supervisada de la carrera de Licenciatura en Enfermería, en el Alojamiento Conjunto de un hospital ubicado en un municipio mediano de la Región Central del Estado de Rio Grande do Sul. La asistencia brindada a la puérpera aún debe ocurrir en el ámbito hospitalario, es decir, en el Alojamiento Conjunto, donde se reconocen los primeros cambios, como el estrés laboral, el dolor, el inicio del proceso de lactancia, el miedo, la inseguridad, la dependencia y los sentimientos de ambivalencia. El objetivo de este trabajo fue reportar la experiencia de una enfermera durante su formación académica, sobre la importancia del cuidado de enfermería puerperal desarrollado en el Alojamiento Conjunto dirigido al cuidado del binomio madre / hijo.

Keywords: Enfermería; Alojamiento conjunto; Amamantamiento.

\section{Introdução}

O Alojamento Conjunto (AC) é o lugar em que a mulher e o recém-nascido (RN) sadio, após o nascimento, permanecem em tempo integral juntos, até a alta hospitalar. Tem o propósito da integração mais próxima da mãe com o RN, favorecendo para: construir um relacionamento afetivo positivo entre o binômio mãe/filho, a partir do nascimento; preparar a mãe e o pai, fortalecendo habilidades e proporcionando segurança emocional m relação aos cuidados com o bebê; estimular o aleitamento materno (AM); minimizar a incidência de infecções hospitalares cruzadas; melhorando integração e avaliação sobre o comportamento natural do binômio (Ministério da Saúde 1982; Ministério da Saúde 2016).

O Ministério da Saúde (2001), assegura que por consequência das modificações, transformações e adaptações que a mulher vivência durante o puerpério, é de extrema importância que seja prestada uma assistência focada em uma atenção mais individualizada e singular para essas usuárias, reconhecendo as particularidades e a identidade de cada puérpera, prestando assim o atendimento humanizado à mulher neste período de maneira integral.

Para que haja promoção da saúde do binômio mãe/filho, é preconizado, sempre que possível, que os mesmos sejam admitidos e continuem no alojamento conjunto por, pelo menos, 48 horas. É fundamental que o profissional de saúde proporcione momentos para a aprendizagem das mães e seus acompanhantes durante a sua permanência e investigue o contexto familiar (Ministério da Saúde, 2012). Investigar e reconhecer o contexto familiar, é relevante para que o profissional possa adequar as suas orientações e condutas baseadas na realidade mais próxima possível das puérperas, alcançando assim, maior aceitação e seguimento destas orientações.

O enfermeiro possui grande destaque como agente multiplicador no tocante das orientações realizadas às puérperas sobre os cuidados prestados ao RN. Transforma-se em um momento de criação de vínculos, com intuito de promover uma assistência de qualidade ao binômio. Evidenciou-se os cuidados fundamentais ofertados ao RN sendo os mais predominantes: a importância do AM, educação em saúde, promoção de vínculos, promoção da saúde, cuidados com o coto umbilical e higiene do RN (Silva, et al., 2017).

O AM é reconhecido pelo Ministério da Saúde, em consenso com a Organização Mundial de Saúde (OMS) e Fundo das Nações Unidas para a Infância (UNICEF), como uma das práticas essenciais para a redução dos índices de mortalidade neonatal. É indispensável o fato de que o AM deve ter início logo após o parto visto que o colostro é apontado com a primeira imunização do RN pela presença de imunoglobulinas e maior quantidade de proteínas e vitamina A. À partir disso, recomendase o AM de forma exclusiva até o sexto mês de vida, e posteriormente e maneira complementar até os dois anos de idade, ou mais (Ministério da Saúde 2011; Oddy 2013; Bezerra et al 2012).

Um dos benefícios de AC está na possibilidade do profissional de enfermagem estimular o fortalecimento do autocuidado e dos cuidados com RN, através de atividades de educação em saúde desenvolvidas durante a assistência prestada no período de internação do binômio mãe/filho. (Brasil, 2016). Levando em considerações as recomendações dos Órgãos de 
Saúde à respeito da importância do AM, é imprescindível que a assistência prestada AC envolva orientações e auxílio a puérpera na prática de amamentação.

Desta maneira, objetivou-se com esse trabalho relatar a experiência de uma enfermeira durante sua formação acadêmica, sobre a importância da assistência de enfermagem puerperal desenvolvida no AC direcionada no cuidado do binômio mãe/filho.

\section{Metodologia}

Trata-se de um relato de experiência desenvolvido durante as práticas da disciplina de Estágio Supervisionado I, do curso de graduação em enfermagem, no Alojamento Conjunto de um hospital localizado em um município de médio porte na região Central do Estado do Rio Grande do Sul. Essas práticas aconteceram entre os meses de março a julho de 2019, envolvendo todas as puérperas atendidas no Sistema Único de Saúde (SUS), durante o pós parto imediato.

Um estudo de caso ou relato de experiência é uma análise e descrição, feita de maneira mais detalhada possível, de alguma situação que apresente alguma particularidade que o torna especial (Pereira, et al 2018).

As visitas após o parto foram realizadas para todas as puérperas internadas no AC, independentemente das faixasetárias e tipo de parto. O objetivo das visitas era realizar em um primeiro momento o acolhimento com escuta qualificada a fim de criar um vínculo de confiança e um ambiente confortável para a puérpera, RN e acompanhante, que na maior parte das internações, era um familiar. A importância do acolhimento e criação do vínculo de confiança com as usuárias era para que estas e seus familiares, juntamente com o RN pudessem se sentir acolhidos e valorizados dentro do Sistema Único de Saúde (SUS).

As visitas foram realizadas no pós parto durante o período mencionado, com uma média de três avaliações diárias, após a admissão da puérpera e RN no AC. Após a avaliação, anamnese e exame físico completo, era realizada uma escuta qualificada a respeito das dúvidas e inseguranças sobre o AM, após as orientações realizadas e explanação das dúvidas de cada puérpera, era proporcionado um momento de prática da amamentação, no qual era auxiliado a puérpera e mostrava-se como era a técnica correta, com o RN posicionado para ser amamentado e, nestes momentos, era possível identificar quais as maiores fragilidades e dificuldades das mesmas, e a partir disso as mesmas eram auxiliadas e orientadas sobre o AM.

Dentre as visitas realizadas, a maior parte das puérperas relatavam dúvidas e inseguranças acerca da prática do AM durante o pós parto imediato e puerpério. Algumas puérperas demonstravam certa resistência e falsas crenças em relação ao leite materno. Nestes momentos, era possível identificar a importância de ser trabalhado este tema e orientado as puérperas desde a gestação, durante o pré-natal, para que no momento do pós-parto, as mesmas já estejam informadas e engajadas sobre essa prática, sendo assim alcançada maior efetividade e menor inseguranças nesses momentos.

Através dessas visitas com acolhimento e avaliação, foi possível a criação de um vínculo de segurança e ambiente agradável para os envolvidos. Todas as dúvidas foram ouvidas e explanadas com orientações que foram para além das dúvidas trazidas, proporcionando assim uma maior proteção e promoção AM durante os primeiros 6 meses de vida do RN. Ficou evidente a importância desta primeira visita, para que após a explanação de dúvidas, fosse possível realizar a prática da amamentação com auxílio profissional para que esse momento pudesse se tornar o mais agradável e seguro possível e com isso, incentivando e engajando a puérpera para a continuidade da prática no domicílio.

\section{Resultados e Discussão}

O início da assistência prestada à puérpera precisa acontecer ainda no ambiente hospitalar, isto é, no AC, onde se reconhecem as primeiras modificações como estresse do parto, dores, início do processo de amamentação, medo, insegurança, dependência e sentimentos de ambivalência (Oliveira, Quirino \& Rodrigues, 2012). 
Dessa forma, torna-se evidente a relevância da assistência de enfermagem no período puerperal no que diz respeito ao processo educativo com intuito de realizar as orientações à mulher para alcançar a autonomia no seu autocuidado e segurança no cuidado com o seu RN no domicílio, o que auxilia na adaptação à nova dinâmica familiar (Garcia, Leite \& Nogueira, 2013).

Foi comprovado o contentamento das puérperas ao auxílio e as orientações prestadas pelos profissionais enfermeiros no AC. É importante reforçar que o intuito da educação em saúde no AC, não é o de impor o conhecimento, nem o de sobrecarregar a puérpera com tantas informações e novas adaptações. (Strefling et al. 2017) Dessa maneira, o profissional possibilite e torne esse momento, um momento de troca de conhecimentos, valorizando o papel desta enquanto mulher e puérpera, buscando realizar as orientações de enfermagem o mais próximo possível da sua realidade. As puérperas precisam receber o incentivo positivo para realizar o autocuidado e prestar cuidados ao seu filho.

Ribeiro et al. (2018), evidenciou que as puérperas possuíam ainda, errôneas concepções a respeito do AM e aos cuidados gerais com o RN. Neste estudo, $40 \%$ das puérperas acreditavam não haver problemas ao realizar a amamentação cruzada. Observou-se o a falta de conhecimento sobre a forma correta da pega do mamilo pelo RN, o que pode estar relacionado ao fato de não haver as orientações, no AC e também durante as consultas de pré-natal. Neste estudo, ainda, 18\% das puérperas não obtiveram informações sobre as técnicas a fim de prevenir as intercorrências mamárias.

A amamentação exclusiva até os seis meses acarreta inúmeras vantagens para o bebê e a mãe. Dentre estas, a primordial delas é a proteção contra infecções gastrointestinais. O começo precoce do AM, dentro de 1 hora após o nascimento, protege o RN de desenvolver infecções e reduz a mortalidade neonatal. O risco de mortalidade por consequência de diarreia e outras infecções pode aumentar em bebês que são parcialmente amamentados ou que não amamentaram. O leite materno também é uma ferramenta fundamental de energia e nutrientes para crianças de 6 a 23 meses. Pode fornecer metade ou mais das necessidades de energia de uma criança entre as idades de 6 e 12 meses, e um terço das necessidades de energia entre 12 e 24 meses (OMS, 2016).

Percebe-se ainda, que o AM é definitivamente, o melhor alimento para a criança, considerado como o primeiro estilo de vida saudável que refletirá seus benefícios até a fase adulta. Portanto é por meio dele que o bebê estará seguramente bem nutrido na fase inicial de sua vida e com o sistema imunológico, fortalecendo-se gradativamente para, assim, o proteger de patologias futuras. Os benefícios não se limitam as crianças, uma vez que a mãe também é protegida de patologias tão comuns como câncer de mama e de útero. Faz-se necessário destacar também importância da equipe de saúde, visando minimizar o problema do desmame precoce (Feitosa, Silva \& Silva 2020).

No leite estão presentes anticorpos que a mãe adquiriu por meio de contato com patógenos, que por sua vez serão transferidos para o bebê, fortalecendo assim o sistema imune do neonato. Vale salientar que mesmo o AM quando exclusivo não substitui a vacinação, visto que esta é essencial para o sistema imune, agindo de diversas formas, entre elas, potencializando os anticorpos já presentes no organismo do bebê. Podemos constatar a importância do AM na construção da imunidade do bebê em particular sua imunidade inata (Silva et al, 2020).

A maneira na qual os enfermeiros referem-se a amamentação com as puérperas, quando praticada de forma efetiva, auxilia para o incentivo ao AM exclusivo. Por essa razão, a valorização da associação da teoria e a prática no que diz respeito a promoção do AM, usufruindo das relações de vínculo, acolhimento, escuta qualificada, aconselhamento e orientações, e os saberes estruturados na assistência ao binômio mãe/filho e no decurso educativo, com objetivo de ofertar o apoio, suporte, orientações necessárias e auxílio para a prática da amamentação (Silva, et al 2014).

Além de focar nos fatores biológicos que condicionam a interrupção da amamentação, o enfermeiro deve estar apto a perceber questões psicológicas e sociais, e desta forma tentar ajudar a mulher a prosseguir com essa prática. A mãe deve sentir confiança no profissional, pois só assim ela irá se abrir com mais facilidade e relatar seus problemas (Feitosa, Silva, Silva 2020). 
O AM é uma prática natural de vínculo, afeto, proteção e nutrição para a criança e constitui a mais sensível, completa, econômica e efetiva ferramenta para a redução da morbimortalidade infantil, sendo recomendada de maneira exclusiva nos primeiros seis meses de vida da criança e seguidamente, complementada até os dois anos de idade. Preconiza-se também que o início da a amamentação ao seio materno seja ainda na primeira hora de vida da criança, e a partir de então, em livre demanda (Ministério Da Saúde, 2015).

Essa prática, mesmo que haja todas as evidências científicas que evidenciam as vantagens e superioridade da amamentação, os esforços de organizações nacionais e internacionais, dos programas e políticas brasileiras de apoio e estimulo ao AM, no Brasil ainda está abaixo do que está preconizado nas recomendações internacionais e do Ministério da Saúde (Ministério Da Saúde, 2015).

Foi identificado discursos, discursos das mulheres opostos da enfermeira e do médico no momento do pré-natal, comprovando uma descontinuidade da assistência e fragilidade de uma escuta qualificada durante as consultas. Esta atuação vai ao encontro para a prática de uma conduta impositiva, na qual o profissional "dono do saber científico" decide o que fazer e o melhor caminho a seguir (Reis, et al 2017). Sendo assim, quando o profissional realiza suas orientações sem levar em consideração a realidade e as crenças a respeito do AM, essas orientações correm risco de serem em vão, pois para a eficácia destas, é necessário haver uma troca de conhecimentos e valorização dos saberes da puérpera.

As puérperas, desde a gestação carregam consigo suas próprias experiências, medos, inseguranças e incertezas e o profissional de saúde dispõe sua visão sobre os acontecimentos envolvidos com a gestação e o parto. Esse momento é apontado pela hegemonia do profissional, constituindo forte influência na escolha (Pires, et al 2010). Então, quando o profissional proporciona para a puérpera um momento de acolhimento, escuta qualificada e troca de conhecimentos, as orientações sobre o AM são mais eficazes e compreensíveis, alcançando assim, mais proteção e promoção do AM.

O AM segue sendo um grande desafio, visto que, o índice nas diversas regiões brasileiras continua abaixo do estipulado pela OMS e Ministério da Saúde que é de amamentação exclusiva até o sexto mês de vida. Estudos comprovam que as condições sociais maternas influenciam diretamente no desmame, já que as jovens ou adolescentes são as que amamentam durante menos tempo, bem como, aquelas que não têm companheiro e/ou apresentam baixa renda (Barbieri, et al 2015).

Neste relato, quase a totalidade das mulheres, contaram ter recebido orientações sobre AM na maternidade. Tem sido referido que neste momento é de grande importância tal prática, pois posterior ao nascimento do RN, a puérpera irá iniciar a prática de amamentação, o que beneficia intervenções de acordo com as necessidades de cada uma. A primeira semana de vida é o período ideal para incentivar e auxiliar a puérpera nas dificuldades com o AM, protegendo e promovendo assim, o AM exclusivo até o $6^{\circ}$ mês de vida (Souza et al., 2011).

A amamentação mesmo que seja um ato natural, é também um ato comportamental, e como tal, pode ser compreendido e aprendido. Logo, é necessário que os profissionais da saúde encorajem, apoiem e auxiliem as puérperas para que estas iniciem e mantenham a amamentação exclusiva durante os primeiros seis meses da criança e que no momento correto, introduza, a alimentação complementar de maneira adequada (Barbieri, et al 2015).

O estabelecimento do acolhimento com comunicação e criação de vínculo, são tecnologias relacionais que sinalizam para os profissionais da saúde o resgate destas práticas também no cenário hospitalar. A educação em saúde reforça os espaços emancipatórios do cuidado. Quando o enfermeiro reconhece os saberes do outro, possibilita as bases humanísticas para pensar no cuidado em sua plenitude. (Savieto, Leão 2016). Sendo assim, é ideal acolher e ouvir a puérpera em seus saberes, dúvidas, angustias e medos, para que com isso, a mesma sinta-se acolhida, valorizada e engajada para praticar a amamentação com segurança e prazer. 
O prática da amamentação não possui vantagens somente para o bebê, mas também para a mãe. Ela auxilia na involução uterina, minimizando o sangramento no pós-parto, além de reduzir as chances de desenvolver câncer de mama e de ovários, também são comprovados benefícios físicos e psicológicos (Brasil, 2017).

A carência de informações e assistência desqualificada refletem diretamente na eficácia da amamentação, favorecendo muitas vezes o desmame precoce, acarretando prejuízos nutricionais e até mesmo intelectuais para a criança. A assistência do profissional de enfermagem associado a amamentação tem como intuito orientar, explanar dúvidas, incentivar, apoiar, além de principalmente ter empatia por cada situação, respeitando a singularidade de cada mulher (Lopes, et al 2020).

Conforme Santos et al (2016), o AM pode ser afetado por consequência da falta de informações necessárias sobre o processo de aleitar. Os primeiros dias pós-parto é o momento em que a puérpera encontra-se mais vulnerável a aceitar opiniões de terceiros, e segui-las caso não tenha sido orientada corretamente sobre a importância da realização desta prática de maneira exclusiva até o sexto mês de vida, reforçando a eficácia do leite materno no que diz respeito a suprir as demandas nutricionais do RN neste período.

A realização da prática de Educação em Saúde tem sido cada vez mais reforçada pelas boas repercussões trazidas, e por não trabalhar apenas com o processo de adoecimento, mas essencialmente com a prevenção, fazendo-o refletir sobre o estilo de vida adotado e sobre quais decisões tomar diante de possíveis dúvidas (Silva, 2017). Quando esta é realizada no AC, torna-se uma ferramenta importante para orientar e reforçar a importância da continuidade da amamentação no domicilio, reforçando as vantagens tanto para o RN quanto para mãe.

Tão importante quanto as orientações realizadas durante o pré-natal, é também no pós-parto, preferencialmente no AC, que é o local onde geralmente começam surgir as dificuldades e inseguranças por estar começando as experiências em uma nova realidade na vida, ocasionando momentos de ansiedade, fragilidade emocional e angustias (Lopes, et al 2020). Nesse momento, as orientações de enfermagem tornam-se eficazes e efetivas quando realizadas com responsabilidade e embasamento teórico, comprovando as vantagens do AM.

Durante o atendimento hospitalar, o enfermeiro precisa focar na prevenção de complicações físicas e também emocionais, para que essa puérpera se sinta preparada, orientada e pronta para vivenciar o período de pós-parto de maneira que a torne protagonista desse processo (Strefling, et al 2017). É importante reforçar, que, a qualquer dúvida surgida ou insegurança durante a praticado AM, pode recorrer e pedir auxílio e orientações ao enfermeiro, para que assim haja proteção e prevenção do AM, interrompendo o desmame precoce a perimira dificuldade encontrada.

Mercado et al. (2017), discute-se sobre os cuidados e orientações de enfermagem as puérperas no AC, comprovando que pode-se dizer que o AC é uma continuidade do pré-natal em relação as orientações. Das orientações referidas nesse relato, o AM exclusivo por 6 meses e o banho de sol, foram as menos citadas pelos profissionais. Comprovando assim uma lacuna, pois as orientações como os cuidados com o RN, amamentação e o autocuidado são de extrema importância na nova realidade que a família irá vivenciar, pois na maior parte das vezes as puérperas sentem medo, insegurança e angústia com as novas experiências vivenciadas (Mercado, et al 2017).

\section{Conclusão}

Através das experiências vivenciadas, foi possível comprovar que as orientações ofertadas pela equipe de enfermagem à puérpera no contexto do $\mathrm{AC}$, auxiliam para a autonomia e a compreensão da puérpera em relação ao $\mathrm{AM}$, sua importância, suas vantagens e evidencias que comprovam sua eficácia quando ofertado de maneira exclusiva até os 6 meses de vida do RN.

Ainda há muitos mitos e crenças em relação ao leite materno e amamentação exclusiva nos primeiros 6 meses de vida do RN, o que torna extremamente necessário o engajamento dos profissionais da saúde no tocante a prevenção e promoção do AM para melhorar a qualidade de vida enquanto bebê e também sobre seus reflexos na vida adulta posteriormente. O curto 
período de permanência da puérpera em uma maternidade, não asseguram o esclarecimento de todas as dúvidas em relação ao pós-parto. Desta maneira, aconselha-se a importância de que a mulher seja orientada e preparada para as questões do puerpério ainda enquanto gestante, no pré-natal, para garantir maior efetividade nas orientações durante a internação no AC.

Considera-se extremamente importante promoção da prática do AM de maneira exclusiva até os primeiros 6 meses de vida do bebê, e posteriormente complementado até, pelo menos os 2 anos de idade, como preconizado pelos Órgãos de Saúde. O AM implica positivamente na saúde e qualidade de vida da criança, desde seu nascimento, até a vida adulta, em virtude disso, é importante que o enfermeiro, esteja engajado e comprometido em orientar e preparar as gestantes e puérperas, no pósparto, para realização da amamentação de maneira efetiva.

Levando em consideração a importância desta temática, sugere-se novos estudos abordando esse tema, para que assim haja maior esclarecimento sobre a relevância da amamentação em todos os aspectos.

\section{Referências}

Bezerra, V. L. V. A. et al. (2012). Aleitamento materno exclusivo e fatores associados a sua interrupção precoce: estudo comparativo entre 1999 e 2008. Rev Paul Pediatr, 30 (2).

Brasil. Ministério da Saúde. (2017). Secretaria de Atenção à Saúde. Departamento de Ações Programáticas Estratégicas. Bases para a discussão da Política Nacional de Promoção, Proteção e Apoio ao Aleitamento Materno.

Brasil. Ministério da Saúde. Portaria n. 2.068, de 21 de outubro de 2016. Institui diretrizes para a organização da atenção integral e humanizada à mulher e ao recém-nascido no Alojamento Conjunto.

Feitosa, M. E. B., Silva, S. E. O., \& Silva, L. L. (2020). Aleitamento materno: causas e consequências do desmame precoce. Research, Society and Development, 9 (7).

Garcia, E. S. G. F., Leite, E. P. R. C., \& Nogueira, D. A. (2013) Assistência de enfermagem às puérperas em unidades de atenção primária. Rev Enferm UFPE online, 7 (10).

Lopes, A. A. S. et al (2020). Percepção das puérperas acerca das orientações de enfermagem quanto ao aleitamento materno. Braz. J. of Develop, 6 (7).

Mercado, N. C. et al. (2017). Cuidados e orientações de enfermagem às puérperas no alojamento conjunto. Revenferm UFPE online, 11 (9).

Ministério da saúde (2001). Parto, aborto e puerpério: assistência humanizada à mulher. Brasília (DF).

Ministério da Saúde (BR) (2011). Secretaria de Políticas da Saúde. Área Técnica da Criança. Atenção humanizada ao recém-nascido de baixo peso - Método Canguru: manual do curso. Brasília: Ministério da Saúde.

Ministério da Saúde (BR). I Encontro Nacional sobre Alojamento Conjunto. Relatório Final. Brasília: Ministério da Saúde/INAN; 1982.

Ministério da Saúde (BR). Secretaria de Atenção à Saúde. Departamento de Atenção Básica. Saúde da criança: aleitamento materno e alimentação complementar. (2a ed.), Brasília: Ministério da Saúde, 2015.

Ministério da Saúde. (2012). Secretaria de Atenção a Saúde. Departamento de Ações Programáticas e Estratégicas. Atenção à saúde do recém-nascido: guia para os profissionais de saúde: cuidados gerais. (2a ed.), Brasília: Ministério da Saúde, (1).

Oddy, W. H. (2013). Breastfeeding in the first hour of life protects against neonatal mortality. J Pediatr, 89 (2).

Oliveira, J. F. B., Quirino, G. S., \& Rodrigues, P. (2012) Percepção das puérperas quanto aos cuidados prestados pela equipe de saúde no puerpério. Rev Rene, $12(1)$.

Organização mundial da saúde (OMS) (2016). Breastfeeding: A Key to Sustainable Development.

Pereira, R. T. A., \& Ferreira, V. A. (2014) Consulta de enfermagem na estratégia Saúde da família. Rev UNIARA, 17 (1).

Pires, D. et al (2010). Influência da assistência profissional em saúde na escolha do tipo de parto: um olhar sócio antropológico na saúde suplementar brasileira. Rev. Bras. Saude Mater. Infantil.

Reis, C. C. et al. (2017). Percepção das mulheres sobre a experiência do primeiro parto: implicações para o cuidado de enfermagem. Ciencia Y Enfermeria XXIII (2).

Ribeiro, S. C. S. S. et al. (2018). Atividade educativa para a promoção do cuidado com o recém-nascido. Saúde e Pesquisa, 11 (3).

Santos, A. N. et al. (2016). Vivência das puérperas nutrizes frente à prática do aleitamento materno. Rev Enferm UFSM, 6 (2).

Savieto, R. M., \& Leão, E. R. (2016). Nursing assistance and Jean Watson: a reflection on empathy. Esc Anna Nery, 20 (1).

Silva, B. T. O., et al. (2017) Cuidados de Enfermagem ao Recém-Nascido no Alojamento Conjunto: uma Revisão Integrativa. UNIT Universidade Tiradentes. 
Research, Society and Development, v. 10, n. 1, e57410112266, 2021

(CC BY 4.0) | ISSN 2525-3409 | DOI: http://dx.doi.org/10.33448/rsd-v10i1.12266

Silva, E. C., et al. (2017). Puerpério e assistência de enfermagem: percepção das mulheres. Rev enferm UFPE online, 11 (7).

Silva, N.M et al. (2014). Conhecimento de puérperas sobre amamentação exclusiva. Rev Bras Enfermagem, 67 (2).

Silva, D. I. S., et al. (2020). A importância do aleitamento materno na imunidade do recém-nascido. Research, Society and Development, 9 (7).

Souza, M. H. N., et al. (2011). Estratégia acolhimento mãe-bebê: aspectos relacionados à clientela atendida em uma unidade básica de saúde do município do Rio de Janeiro. Escola Anna Nery, 15 (4).

Strefling, I. S. S., et al. (2017). Percepções de puérperas sobre o cuidado de enfermagem no alojamento conjunto. Res.: Fundam. Care. Online, 9 (2).

Strefling, I. S. S., et al. (2017). Percepções de puérperas sobre o cuidado de enfermagem no alojamento conjunto. Revista de Pesquisa: Cuidado é Fundamental Online, 9 (2). 